\title{
Transversality Conditions for Infinite Horizon Variational Problems on Time Scales*
}

\author{
Agnieszka B. Malinowska ${ }^{1,2} \quad$ Natália Martins $^{1}$ \\ abmalinowska@ua.pt \\ natalia@ua.pt \\ Delfim F. M. Torres ${ }^{1}$ \\ delfim@ua.pt \\ ${ }^{1}$ Department of Mathematics \\ ${ }^{2}$ Faculty of Computer Science \\ University of Aveiro \\ 3810-193 Aveiro, Portugal \\ Białystok University of Technology \\ 15-351 Białystok, Poland
}

\begin{abstract}
We consider problems of the calculus of variations on unbounded time scales. We prove the validity of the Euler-Lagrange equation on time scales for infinite horizon problems, and a new transversality condition.
\end{abstract}

Keywords: time scales, calculus of variations, Euler-Lagrange equation, transversality condition, infinite horizon.

Mathematics Subject Classification 2010: 49K15, 34N05, 39A12.

\section{Introduction}

Starting with Ramsey's pioneering work 31, infinite horizon variational and optimal control problems have been widely used in economics (see, e.g., [6, 13, 14, 15, 16, 17, 20, 30]). On the other hand, economics is a discipline in which there appears to be many opportunities for applications of time scales [1, 2, 3, 4, 25. Therefore, it is natural to try to relate the subject of time scales with the study of infinite horizon variational problems. This is the main motivation and goal of the present work.

The study of improper integrals on time scales was introduced by Bohner and Guseinov in 9 . For a more general treatment see 24. However, the use of time scale integrals in the calculus of variations has been, so far, restricted to bounded intervals - see [5, 7, 8, 18, 26, 27] and references therein. In this paper we consider the infinite horizon problem of maximizing the expression

$$
\int_{a}^{T} L\left(t, x^{\sigma}(t), x^{\Delta}(t)\right) \Delta t
$$

as $T$ grows to infinity. If $T=+\infty$, then the integral (1) does not necessarily converge. It may diverge to plus or minus infinity or it may oscillate. In such situations the extension of the definition of optimality used in the time scale setting (see [7) to the unbounded time domain is not very useful. For example, if every admissible function $x$ yields an infinite value for functional (1), then each admissible path could be called an optimal path. To handle this and similar situations in a rigorous way, several alternative definitions of optimality for problems with unbounded time domain have been proposed in the literature (see, e.g., [12, 19, 32, 33]). In this paper we follow Brock's notion of optimality. Therefore, our optimality criterion for the special case $\mathbb{T}=\mathbb{Z}$ coincides with Brock's notion of weak maximality [12]. If $\mathbb{T}=\mathbb{R}$, our definition of weak maximality coincides with the extension of Brock's notion of weak maximality to the continuous time situation 222. It is worth to point out that in the case where the functional (1) converges for all admissible paths, the weak maximal path is optimal in the sense of the standard definition of optimality.

Main result of the paper gives necessary conditions of weak maximality for infinite horizon variational problems on a generic (unbounded) time scale (cf. Theorem 44).

*Submitted 6-October-2009; Accepted 19-March-2010 in revised form; for publication in Optimization Letters. 


\section{Preliminaries}

In this section we introduce basic definitions and results that will be needed in the sequel. For a more general presentation of the theory of time scales we refer the reader to the books [10, 11]. As usual, $\mathbb{R}, \mathbb{Z}$, and $\mathbb{N}$ denote, respectively, the set of real, integer, and natural numbers.

A time scale $\mathbb{T}$ is an arbitrary nonempty closed subset of $\mathbb{R}$. Besides standard cases of $\mathbb{R}$ (continuous time) and $\mathbb{Z}$ (discrete time), many different models of time are used. For each time scale $\mathbb{T}$ the following operators are used:

- the forward jump operator $\sigma: \mathbb{T} \rightarrow \mathbb{T}, \sigma(t):=\inf \{s \in \mathbb{T}: s>t\}$ for $t \neq \sup \mathbb{T}$ and $\sigma(\sup \mathbb{T})=\sup \mathbb{T}$ if $\sup \mathbb{T}<+\infty$

- the backward jump operator $\rho: \mathbb{T} \rightarrow \mathbb{T}, \rho(t):=\sup \{s \in \mathbb{T}: s<t\}$ for $t \neq \inf \mathbb{T}$ and $\rho(\inf \mathbb{T})=\inf \mathbb{T}$ if $\inf \mathbb{T}>-\infty$;

- the forward graininess function $\mu: \mathbb{T} \rightarrow[0, \infty[, \mu(t):=\sigma(t)-t$.

Example 1. If $\mathbb{T}=\mathbb{R}$, then for any $t \in \mathbb{R}, \sigma(t)=t=\rho(t)$ and $\mu(t) \equiv 0$. If $\mathbb{T}=\mathbb{Z}$, then for every $t \in \mathbb{Z}, \sigma(t)=t+1, \rho(t)=t-1$ and $\mu(t) \equiv 1$.

A point $t \in \mathbb{T}$ is called right-dense, right-scattered, left-dense and left-scattered if $\sigma(t)=t$, $\sigma(t)>t, \rho(t)=t$, and $\rho(t)<t$, respectively. We say that $t$ is isolated if $\rho(t)<t<\sigma(t)$, that $t$ is dense if $\rho(t)=t=\sigma(t)$. If $\sup \mathbb{T}$ is finite and left-scattered, we define $\mathbb{T}^{\kappa}:=\mathbb{T} \backslash\{\sup \mathbb{T}\}$. Otherwise, $\mathbb{T}^{\kappa}:=\mathbb{T}$.

Definition 1. Let $f: \mathbb{T} \rightarrow \mathbb{R}$ and $t \in \mathbb{T}^{\kappa}$. The delta derivative of $f$ at $t$ is the real number $f^{\Delta}(t)$ with the property that given any $\varepsilon$ there is a neighborhood $U$ of $t$ (i.e., $U=] t-\delta, t+\delta[\cap \mathbb{T}$ for some $\delta>0$ ) such that

$$
\left|(f(\sigma(t))-f(s))-f^{\Delta}(t)(\sigma(t)-s)\right| \leq \varepsilon|\sigma(t)-s|
$$

for all $s \in U$. We say that $f$ is delta differentiable on $\mathbb{T}$ provided $f^{\Delta}(t)$ exists for all $t \in \mathbb{T}^{\kappa}$.

We shall often denote $f^{\Delta}(t)$ by $\frac{\Delta}{\Delta t} f(t)$ if $f$ is a composition of other functions. The delta derivative of a function $f: \mathbb{T} \rightarrow \mathbb{R}^{n}(n \in \mathbb{N})$ is a vector whose components are delta derivatives of the components of $f$. For $f: \mathbb{T} \rightarrow X$, where $X$ is an arbitrary set, we define $f^{\sigma}:=f \circ \sigma$.

For delta differentiable $f$ and $g$, the next formulas hold:

$$
\begin{aligned}
f^{\sigma}(t) & =f(t)+\mu(t) f^{\Delta}(t), \\
(f g)^{\Delta}(t) & =f^{\Delta}(t) g^{\sigma}(t)+f(t) g^{\Delta}(t) \\
& =f^{\Delta}(t) g(t)+f^{\sigma}(t) g^{\Delta}(t) .
\end{aligned}
$$

Remark 1 . If $\mathbb{T}=\mathbb{R}$, then $f: \mathbb{R} \rightarrow \mathbb{R}$ is delta differentiable at $t \in \mathbb{R}$ if and only if $f$ is differentiable in the ordinary sense at $t$. Then, $f^{\Delta}(t)=f^{\prime}(t)$. If $\mathbb{T}=\mathbb{Z}$, then $f: \mathbb{Z} \rightarrow \mathbb{R}$ is always delta differentiable at every $t \in \mathbb{Z}$ with $f^{\Delta}(t)=f(t+1)-f(t)$.

Let $a, b \in \mathbb{T}, a<b$. We define the interval $[a, b]$ in $\mathbb{T}$ by

$$
[a, b]:=\{t \in \mathbb{T}: a \leq t \leq b\} .
$$

Open intervals, half-open intervals and unbounded intervals in $\mathbb{T}$ are defined accordingly.

Definition 2. A function $F: \mathbb{T} \rightarrow \mathbb{R}$ is called a delta antiderivative of $f: \mathbb{T} \rightarrow \mathbb{R}$ provided

$$
F^{\Delta}(t)=f(t), \quad \forall t \in \mathbb{T}^{\kappa} .
$$

In this case we define the delta integral of $f$ from $a$ to $b(a, b \in \mathbb{T})$ by

$$
\int_{a}^{b} f(t) \Delta t:=F(b)-F(a) .
$$


In order to present a class of functions that possess a delta antiderivative, the following definition is introduced:

Definition 3. A function $f: \mathbb{T} \rightarrow \mathbb{R}$ is called $r d$-continuous if it is continuous at the right-dense points in $\mathbb{T}$ and its left-sided limits exist at all left-dense points in $\mathbb{T}$. A function $f: \mathbb{T} \rightarrow \mathbb{R}^{n}$ is $r d$-continuous if all its components are rd-continuous.

The set of all rd-continuous functions $f: \mathbb{T} \rightarrow \mathbb{R}^{n}$ is denoted by $\mathrm{C}_{r d}\left(\mathbb{T}, \mathbb{R}^{n}\right)$, or simply by $\mathrm{C}_{r d}$. Similarly, $\mathrm{C}_{r d}^{1}\left(\mathbb{T}, \mathbb{R}^{n}\right)$ and $\mathrm{C}_{r d}^{1}$ will denote the set of functions from $\mathrm{C}_{r d}$ whose delta derivative belongs to $\mathrm{C}_{r d}$.

Theorem 1 ([10]). Every rd-continuous function has a delta antiderivative. In particular, if $a \in \mathbb{T}$, then the function $F$ defined by $F(t)=\int_{a}^{t} f(\tau) \Delta \tau, t \in \mathbb{T}$, is a delta antiderivative of $f$.

Theorem 2 (10]). If $a, b, c \in \mathbb{T}, a \leq c \leq b, \alpha \in \mathbb{R}$, and $f, g \in C_{r d}(\mathbb{T}, \mathbb{R})$, then

1. $\int_{a}^{b}(f(t)+g(t)) \Delta t=\int_{a}^{b} f(t) \Delta t+\int_{a}^{b} g(t) \Delta t$

2. $\int_{a}^{b} \alpha f(t) \Delta t=\alpha \int_{a}^{b} f(t) \Delta t$;

3. $\int_{a}^{b} f(t) \Delta t=-\int_{b}^{a} f(t) \Delta t$;

4. $\int_{a}^{a} f(t) \Delta t=0$;

5. $\int_{a}^{b} f(t) \Delta t=\int_{a}^{c} f(t) \Delta t+\int_{c}^{b} f(t) \Delta t$

6. If $f(t)>0$ for all $a \leq t<b$, then $\int_{a}^{b} f(t) \Delta t>0$;

\%. $\int_{a}^{b} f^{\sigma}(t) g^{\Delta}(t) \Delta t=[(f g)(t)]_{t=a}^{t=b}-\int_{a}^{b} f^{\Delta}(t) g(t) \Delta t ;$

8. $\int_{a}^{b} f(t) g^{\Delta}(t) \Delta t=[(f g)(t)]_{t=a}^{t=b}-\int_{a}^{b} f^{\Delta}(t) g^{\sigma}(t) \Delta t ;$

9. If $t \in \mathbb{T}^{k}$, then $\int_{t}^{\sigma(t)} f(\tau) \Delta \tau=\mu(t) f(t)$.

Definition 4. If $a \in \mathbb{T}$, sup $\mathbb{T}=+\infty$ and $f \in C_{r d}([a,+\infty[, \mathbb{R})$, then we define the improper delta integral by

$$
\int_{a}^{+\infty} f(t) \Delta t:=\lim _{b \rightarrow+\infty} \int_{a}^{b} f(t) \Delta t
$$

provided this limits exists (in $\overline{\mathbb{R}}:=\mathbb{R} \cup\{-\infty,+\infty\}$ ). We say that the improper delta integral converges if this limit is finite; otherwise, we say that the improper delta integral diverges.

In [9] the reader may find many examples and results involving delta improper integrals.

The following result will be very useful in the proof of our main result (Theorem 41).

Theorem 3 (23]). Let $S$ and $T$ be subsets of a normed vector space. Let $f$ be a map defined on $T \times S$, having values in some complete normed vector space. Let $v$ be adherent to $S$ and $w$ adherent to T. Assume that:

1. $\lim _{x \rightarrow v} f(t, x)$ exists for each $t \in T$;

2. $\lim _{t \rightarrow w} f(t, x)$ exists uniformly for $x \in S$.

Then the limits $\lim _{t \rightarrow w} \lim _{x \rightarrow v} f(t, x), \lim _{x \rightarrow v} \lim _{t \rightarrow w} f(t, x)$, and $\lim _{(t, x) \rightarrow(v, w)} f(t, x)$ all exist and are equal. 


\section{Main results}

In this section we assume that $\mathbb{T}$ is a time scale such that $\sup \mathbb{T}=+\infty$. In what follows we will suppose that $a, T, T^{\prime} \in \mathbb{T}$ are such that $T>a$ and $T^{\prime}>a$. By $\partial_{2} L$ and $\partial_{3} L$ we denote, respectively, the partial derivative of $L(\cdot, \cdot, \cdot)$ with respect to its second and third argument. Let us consider the following variational problem on $\mathbb{T}$ :

$$
\begin{gathered}
\int_{a}^{+\infty} L\left(t, x^{\sigma}(t), x^{\Delta}(t)\right) \Delta t \longrightarrow \max \\
x \in C_{r d}^{1}\left(\mathbb{T}, \mathbb{R}^{n}\right) \\
x(a)=x_{a}
\end{gathered}
$$

where $(u, v) \rightarrow L(t, u, v)$ is a $C^{1}\left(\mathbb{R}^{2 n}, \mathbb{R}\right)$ function for any $t \in \mathbb{T}, \partial_{3} L\left(t, x^{\sigma}(t), x^{\Delta}(t)\right)$ is delta differentiable for all $x \in C_{r d}^{1}\left(\mathbb{T}, \mathbb{R}^{n}\right), n \in \mathbb{N}$, and $x_{a} \in \mathbb{R}^{n}$.

Definition 5. We say that $x$ is an admissible path for problem (2) if and only if $x \in C_{r d}^{1}\left(\mathbb{T}, \mathbb{R}^{n}\right)$ and $x(a)=x_{a}$.

We use the following notion as our optimality criteria.

Definition 6 (weak maximality). We say that $x_{*}$ is weakly maximal to problem (2) if and only if $x_{*}$ is an admissible path and

$$
\lim _{T \rightarrow+\infty} \inf _{T^{\prime} \geq T} \int_{a}^{T^{\prime}}\left[L\left(t, x^{\sigma}(t), x^{\Delta}(t)\right)-L\left(t, x_{*}^{\sigma}(t), x_{*}^{\Delta}(t)\right] \Delta t \leq 0\right.
$$

for all admissible path $x$.

Lemma 1. Let $g \in C_{r d}(\mathbb{T}, \mathbb{R})$. Then,

$$
\lim _{T \rightarrow+\infty} \inf _{T^{\prime} \geq T} \int_{a}^{T^{\prime}} g(t) \eta^{\sigma}(t) \Delta t=0 \quad \text { for all } \eta \in C_{r d}(\mathbb{T}, \mathbb{R}) \quad \text { such that } \eta(a)=0
$$

if and only if $g(t)=0$ on $[a,+\infty[$.

Proof. The implication " $\Leftarrow "$ is obvious. Let us prove the implication " $\Rightarrow "$. Suppose, by contradiction, that $g(t) \not \equiv 0$. Let $t_{0}$ be a point on $\left[a,+\infty\left[\right.\right.$ such that $g\left(t_{0}\right) \neq 0$; suppose, without loss of generality, that $g\left(t_{0}\right)>0$.

Case $I$. If $t_{0}$ is right-dense, then $g$ is also positive in $\left[t_{0}, t_{1}\right]$ for some $t_{1}>t_{0}$. Define

$$
\eta(t)= \begin{cases}\left(t-t_{0}\right)\left(t_{1}-t\right) & t \in\left[t_{0}, t_{1}\right] \\ 0 & \text { otherwise }\end{cases}
$$

Then

$$
\lim _{T \rightarrow+\infty} \inf _{T^{\prime} \geq T} \int_{a}^{T^{\prime}} g(t) \eta^{\sigma}(t) \Delta t=\int_{t_{0}}^{t_{1}} g(t) \eta^{\sigma}(t) \Delta t>0
$$

which is a contradiction.

Case II. Suppose that $t_{0}$ is right-scattered.

1. If $\sigma\left(t_{0}\right)$ is right-scattered, define

$$
\eta(t)=\left\{\begin{array}{ll}
g\left(t_{0}\right) & t=\sigma\left(t_{0}\right) \\
0 & \text { otherwise }
\end{array} .\right.
$$

Then

$$
\lim _{T \rightarrow+\infty} \inf _{T^{\prime} \geq T} \int_{a}^{T^{\prime}} g(t) \eta^{\sigma}(t) \Delta t=\int_{t_{0}}^{\sigma\left(t_{0}\right)} g(t) \eta^{\sigma}(t) \Delta t=\mu\left(t_{0}\right) g\left(t_{0}\right) g\left(t_{0}\right)>0
$$

which is a contradiction. 
2. Suppose that $\sigma\left(t_{0}\right)$ is right-dense. Two situations may occur:

(a) $g\left(\sigma\left(t_{0}\right)\right) \neq 0$;

(b) $g\left(\sigma\left(t_{0}\right)\right)=0$.

In case $(a)$, we can assume, without loss of generality, that $g\left(\sigma\left(t_{0}\right)\right)>0$. Then $g$ is also positive in $\left[\sigma\left(t_{0}\right), t_{2}\right]$ for some $t_{2}>\sigma\left(t_{0}\right)$. Define

$$
\eta(t)=\left\{\begin{array}{ll}
\left(t-\sigma\left(t_{0}\right)\right)\left(t_{2}-t\right) & t \in\left[\sigma\left(t_{0}\right), t_{2}\right] \\
0 & \text { otherwise }
\end{array} .\right.
$$

In this case

$$
\lim _{T \rightarrow+\infty} \inf _{T^{\prime} \geq T} \int_{a}^{T^{\prime}} g(t) \eta^{\sigma}(t) \Delta t=\int_{\sigma\left(t_{0}\right)}^{t_{2}} g(t) \eta^{\sigma}(t) \Delta t>0
$$

which is a contradiction.

Suppose we are in case $(b)$. Two situations may happen:

(i) $g(t)=0$ on $\left[\sigma\left(t_{0}\right), t_{3}\right]$ for some $t_{3}>\sigma\left(t_{0}\right)$;

(ii) $\forall t_{3}>\sigma\left(t_{0}\right) \exists t \in\left[\sigma\left(t_{0}\right), t_{3}\right] g(t) \neq 0$.

In case $(i)$ define

$$
\eta(t)= \begin{cases}g\left(t_{0}\right) & t=\sigma\left(t_{0}\right) \\ \varphi(t) & \left.t \in] \sigma\left(t_{0}\right), t_{3}\right] \\ 0 & \text { otherwise }\end{cases}
$$

for some function $\varphi \in C_{r d}$ satisfying the conditions $\varphi\left(t_{3}\right)=0$ and $\varphi\left(\sigma\left(t_{0}\right)\right)=g\left(t_{0}\right)$. It follows that

$$
\lim _{T \rightarrow+\infty} \inf _{T^{\prime} \geq T} \int_{a}^{T^{\prime}} g(t) \eta^{\sigma}(t) \Delta t=\int_{t_{0}}^{\sigma\left(t_{0}\right)} g(t) \eta^{\sigma}(t) \Delta t=\mu\left(t_{0}\right) g\left(t_{0}\right) g\left(t_{0}\right)>0
$$

which is a contradiction.

Suppose we are in case $(i i)$. Since $\sigma\left(t_{0}\right)$ is right-dense, there exists a strictly decreasing sequence $S=\left\{s_{k}: k \in \mathbb{N}\right\} \subseteq \mathbb{T}$ such that $\lim _{k \rightarrow \infty} s_{k}=\sigma\left(t_{0}\right)$ and $g\left(s_{k}\right) \neq 0, \forall k \in \mathbb{N}$. If there exists a right-dense $s_{k}$, then go to Case $I$ with $t_{0}:=s_{k}$ (and we get a contradiction). If all points of the sequence are right-scattered, then go to Case II with $t_{0}:=s_{j}$ for some $j \in \mathbb{N}$. Since $\sigma\left(t_{0}\right)$ is right-scattered, we are in situation 1 and we obtain a contradiction.

Therefore, we may conclude that $g \equiv 0$ on $[a,+\infty[$.

Theorem 4. Suppose that the optimal path to problem (2) exists and is given by $x_{*}$. Let $p \in$ $C_{r d}^{1}\left(\mathbb{T}, \mathbb{R}^{n}\right)$ be such that $p(a)=0$. Define

$$
\begin{aligned}
& A\left(\varepsilon, T^{\prime}\right):=\int_{a}^{T^{\prime}} \frac{L\left(t, x_{*}^{\sigma}(t)+\varepsilon p^{\sigma}(t), x_{*}^{\Delta}(t)+\varepsilon p^{\Delta}(t)\right)-L\left(t, x_{*}^{\sigma}(t), x_{*}^{\Delta}(t)\right)}{\varepsilon} \Delta t \\
& V(\varepsilon, T):=\inf _{T^{\prime} \geq T} \int_{a}^{T^{\prime}}\left[L\left(t, x_{*}^{\sigma}(t)+\varepsilon p^{\sigma}(t), x_{*}^{\Delta}(t)+\varepsilon p^{\Delta}(t)\right)-L\left(t, x_{*}^{\sigma}(t), x_{*}^{\Delta}(t)\right)\right] \Delta t \\
& V(\varepsilon) \quad:=\lim _{T \rightarrow+\infty} V(\varepsilon, T) .
\end{aligned}
$$

Suppose that

1. $\lim _{\varepsilon \rightarrow 0} \frac{V(\varepsilon, T)}{\varepsilon}$ exists for all $T$; 
2. $\lim _{T \rightarrow+\infty} \frac{V(\varepsilon, T)}{\varepsilon}$ exists uniformly for $\varepsilon$;

3. For every $T^{\prime}>a, T>a$, and $\varepsilon \in \mathbb{R} \backslash\{0\}$, there exists a sequence $\left(A\left(\varepsilon, T_{n}^{\prime}\right)\right)_{n \in \mathbb{N}}$ such that

$$
\lim _{n \rightarrow+\infty} A\left(\varepsilon, T_{n}^{\prime}\right)=\inf _{T^{\prime} \geq T} A\left(\varepsilon, T^{\prime}\right)
$$

uniformly for $\varepsilon$.

Then $x_{*}$ satisfies the Euler-Lagrange equation

$$
\frac{\Delta}{\Delta t} \partial_{3} L\left(t, x^{\sigma}(t), x^{\Delta}(t)\right)=\partial_{2} L\left(t, x^{\sigma}(t), x^{\Delta}(t)\right), \quad \forall t \in[a,+\infty[
$$

and the tranversality condition

$$
\lim _{T \rightarrow+\infty} \inf _{T^{\prime} \geq T} \partial_{3} L\left(T^{\prime}, x^{\sigma}\left(T^{\prime}\right), x^{\Delta}\left(T^{\prime}\right)\right) x\left(T^{\prime}\right)=0 .
$$

Remark 2. Similarly to the classical context $\mathbb{T}=\mathbb{R}[29$, hypotheses 1,2 , and 3 of Theorem 4 are impossible to be verified a priori because $x_{*}$ is unknown. In practical terms such hypotheses are assumed to be true and conditions (3) and (4) are applied heuristically to obtain a candidate. If such a candidate is, or not, a solution to the problem is a different question that always require further analysis (see Examples 2 and 3).

Proof. Using our notion of weak maximality, if $x_{*}$ is optimal, then $V(\varepsilon) \leq 0$ for every $\varepsilon \in \mathbb{R}$. Since $V(0)=0$, then 0 is an extremal point of $V$. If $V$ is differentiable at $t=0$, we may conclude that $V^{\prime}(0)=0$. We now note that

$$
\begin{aligned}
V^{\prime}(0)= & \lim _{\varepsilon \rightarrow 0} \frac{V(\varepsilon)}{\varepsilon}=\lim _{\varepsilon \rightarrow 0} \lim _{T \rightarrow+\infty} \frac{V(\varepsilon, T)}{\varepsilon} \\
= & \lim _{T \rightarrow+\infty} \lim _{\varepsilon \rightarrow 0} \frac{V(\varepsilon, T)}{\varepsilon} \quad \text { by hypothesis 1 and 2 and Theorem 3) } \\
= & \lim _{T \rightarrow+\infty} \lim _{\varepsilon \rightarrow 0} \inf _{T^{\prime} \geq T} A\left(\varepsilon, T^{\prime}\right) \\
= & \lim _{T \rightarrow+\infty} \lim _{\varepsilon \rightarrow 0} \lim _{n \rightarrow+\infty} A\left(\varepsilon, T_{n}^{\prime}\right) \quad \text { (by hypothesis 3) } \\
= & \lim _{T \rightarrow+\infty} \lim _{n \rightarrow+\infty} \lim _{\varepsilon \rightarrow 0} A\left(\varepsilon, T_{n}^{\prime}\right) \quad \text { (by hypothesis 3 and Theorem 3) } \\
= & \lim _{T \rightarrow+\infty} \inf _{T^{\prime} \geq T} \lim _{\varepsilon \rightarrow 0} A\left(\varepsilon, T^{\prime}\right) \quad \text { (by hypothesis 3) } \\
= & \lim _{T \rightarrow+\infty} \inf _{T^{\prime} \geq T} \lim _{\varepsilon \rightarrow 0} \int_{a}^{T^{\prime}} \frac{L\left(t, x_{*}^{\sigma}(t)+\varepsilon p^{\sigma}(t), x_{*}^{\Delta}(t)+\varepsilon p^{\Delta}(t)\right)-L\left(t, x_{*}^{\sigma}(t), x_{*}^{\Delta}(t)\right)}{\varepsilon} \Delta t \\
= & \lim _{T \rightarrow+\infty} \inf _{T^{\prime} \geq T} \int_{a}^{T^{\prime}} \lim _{\varepsilon \rightarrow 0} \frac{L\left(t, x_{*}^{\sigma}(t)+\varepsilon p^{\sigma}(t), x_{*}^{\Delta}(t)+\varepsilon p^{\Delta}(t)\right)-L\left(t, x_{*}^{\sigma}(t), x_{*}^{\Delta}(t)\right)}{\varepsilon} \Delta t \\
= & \lim _{T \rightarrow+\infty} \inf _{T^{\prime} \geq T} \int_{a}^{T^{\prime}}\left[\partial_{2} L\left(t, x_{*}^{\sigma}(t), x_{*}^{\Delta}(t)\right) p^{\sigma}(t)+\partial_{3} L\left(t, x_{*}^{\sigma}(t), x_{*}^{\Delta}(t)\right) p^{\Delta}(t)\right] \Delta t \\
= & \lim _{T \rightarrow+\infty} \inf _{T^{\prime} \geq T}\left\{\int_{a}^{T^{\prime}}\left[\partial_{2} L\left(t, x_{*}^{\sigma}(t), x_{*}^{\Delta}(t)\right) p^{\sigma}(t)-\frac{\Delta}{\Delta t} \partial_{3} L\left(t, x_{*}^{\sigma}(t), x_{*}^{\Delta}(t)\right) p^{\sigma}(t)\right] \Delta t\right. \\
& \left.+\partial_{3} L\left(T^{\prime}, x_{*}^{\sigma}\left(T^{\prime}\right), x_{*}^{\Delta}\left(T^{\prime}\right)\right) p\left(T^{\prime}\right)\right\} \quad(\text { by item } 8 \text { of Theorem 2 and } p(a)=0) .
\end{aligned}
$$

Hence we may conclude that

$$
\lim _{T \rightarrow+\infty} \inf _{T^{\prime} \geq T}\left\{\int_{a}^{T^{\prime}}\left(\partial_{2} L(\bullet)-\frac{\Delta}{\Delta t} \partial_{3} L(\bullet)\right) p^{\sigma}(t) \Delta t+\partial_{3} L\left(T^{\prime}, x_{*}^{\sigma}\left(T^{\prime}\right), x_{*}^{\Delta}\left(T^{\prime}\right)\right) p\left(T^{\prime}\right)\right\}=0
$$

where we denote $(\bullet):=\left(t, x_{*}^{\sigma}(t), x_{*}^{\Delta}(t)\right)$. Since (5) holds for all $p \in C_{r d}^{1}$ such that $p(a)=0$, then, in particular, (5) holds for $p$ satisfying also $p\left(T^{\prime}\right)=0$. Therefore,

$$
\lim _{T \rightarrow+\infty} \inf _{T^{\prime} \geq T} \int_{a}^{T^{\prime}}\left(\partial_{2} L(\bullet)-\frac{\Delta}{\Delta t} \partial_{3} L(\bullet)\right) p^{\sigma}(t) \Delta t=0 .
$$


Denote

$$
\partial_{2} L=\left(\frac{\partial L}{\partial x_{1}}, \cdots, \frac{\partial L}{\partial x_{n}}\right) \quad \text { and } \quad \partial_{3} L=\left(\frac{\partial L}{\partial y_{1}}, \cdots, \frac{\partial L}{\partial y_{n}}\right) .
$$

Choosing $p=\left(p_{1}, \cdots, p_{n}\right)$ such that $p_{2}, \ldots, p_{n} \equiv 0$, we obtain from (6) that

$$
\lim _{T \rightarrow+\infty} \inf _{T^{\prime} \geq T} \int_{a}^{T^{\prime}}\left(\frac{\partial L}{\partial x_{1}}(\bullet)-\frac{\Delta}{\Delta t} \frac{\partial L}{\partial y_{1}}(\bullet)\right) p_{1}^{\sigma}(t) \Delta t=0 .
$$

Using Lemma 1 we conclude that

$$
\frac{\partial L}{\partial x_{1}}(\bullet)-\frac{\Delta}{\Delta t} \frac{\partial L}{\partial y_{1}}(\bullet)=0, \quad \forall t \in[a,+\infty[.
$$

This procedure can be similarly used for the other coordinates and we obtain the Euler-Lagrange equations:

$$
\frac{\partial L}{\partial x_{i}}(\bullet)-\frac{\Delta}{\Delta t} \frac{\partial L}{\partial y_{i}}(\bullet)=0, \quad \forall t \in[a,+\infty[
$$

for $i=2,3, \ldots, n$. These $n$ Euler-Lagrange equations can be written in the condensed form

$$
\partial_{2} L\left(t, x_{*}^{\sigma}(t), x_{*}^{\Delta}(t)\right)-\frac{\Delta}{\Delta t} \partial_{3} L\left(t, x_{*}^{\sigma}(t), x_{*}^{\Delta}(t)\right)=0, \quad \forall t \in[a,+\infty[.
$$

The Euler-Lagrange equation (77) and equation (5) shows that

$$
\lim _{T \rightarrow+\infty} \inf _{T^{\prime} \geq T} \partial_{3} L\left(T^{\prime}, x_{*}^{\sigma}\left(T^{\prime}\right), x_{*}^{\Delta}\left(T^{\prime}\right)\right) p\left(T^{\prime}\right)=0 .
$$

Next we consider a special curve $p$ defined by

$$
p(t)=\alpha(t) x_{*}(t), \quad \forall t \in[a,+\infty[
$$

where $\alpha:\left[a,+\infty\left[\rightarrow \mathbb{R}\right.\right.$ is a $C_{r d}^{1}$ function satisfying $\alpha(a)=0$ and there exists $T_{0} \in \mathbb{T}$ such that $\alpha(t)=\beta \in \mathbb{R} \backslash\{0\}$, for all $t>T_{0}$. By equation (8) we conclude that

$$
\begin{aligned}
0 & =\lim _{T \rightarrow+\infty} \inf _{T^{\prime} \geq T} \partial_{3} L\left(T^{\prime}, x_{*}^{\sigma}\left(T^{\prime}\right), x_{*}^{\Delta}\left(T^{\prime}\right)\right) \alpha\left(T^{\prime}\right) x_{*}\left(T^{\prime}\right) \\
& =\lim _{T \rightarrow+\infty} \inf _{T^{\prime} \geq T} \partial_{3} L\left(T^{\prime}, x_{*}^{\sigma}\left(T^{\prime}\right), x_{*}^{\Delta}\left(T^{\prime}\right)\right) \beta x_{*}\left(T^{\prime}\right)
\end{aligned}
$$

and therefore

$$
\left.\lim _{T \rightarrow+\infty} \inf _{T^{\prime} \geq T} \partial_{3} L\left(T^{\prime}, x_{*}^{\sigma}\left(T^{\prime}\right), x_{*}^{\Delta}\left(T^{\prime}\right)\right)\right) x_{*}\left(T^{\prime}\right)=0,
$$

proving that $x_{*}$ satisfies the transversality condition (4).

\section{Illustrative examples}

Example 2. Consider the problem

$$
\int_{a}^{+\infty}\left[\left(x^{\sigma}(t)-\alpha\right)^{2}+\beta x^{\Delta}(t)\right] \Delta t \longrightarrow \max , \quad x(a)=\alpha,
$$

where $\alpha>0$ and $\beta>0$. Since $L\left(t, x^{\sigma}, x^{\Delta}\right)=\left(x^{\sigma}-\alpha\right)^{2}+\beta x^{\Delta}$ we have $\partial_{2} L=2\left(x^{\sigma}-\alpha\right)$ and $\partial_{3} L=\beta$. From Theorem 4 the Euler-Lagrange equation is

$$
2\left(x^{\sigma}(t)-\alpha\right)=0, \forall t \in[a,+\infty[,
$$

that is, $x^{\sigma}(t)=\alpha, \forall t \in[a,+\infty[$. As $x(a)=\alpha$, we have $x(t)=\alpha, \forall t \in[a,+\infty[$. Observe that the transversality condition (4) is not satisfied, because

$$
\lim _{T \rightarrow+\infty} \inf _{T^{\prime} \geq T} \partial_{3} L\left(T^{\prime}, x^{\sigma}\left(T^{\prime}\right), x^{\Delta}\left(T^{\prime}\right)\right) x\left(T^{\prime}\right)=\beta \alpha>0 .
$$


The reason why we obtain this contradiction is that assumptions of Theorem 4 are violated. Consider $L\left(t, x^{\sigma}(t)+\varepsilon p^{\sigma}(t), x^{\Delta}(t)+\varepsilon p^{\Delta}(t)\right)-L\left(t, x^{\sigma}(t), x^{\Delta}(t)\right)$. Substituting $x(t)=\alpha$ into it, we have

$$
L\left(t, x^{\sigma}(t)+\varepsilon p^{\sigma}(t), x^{\Delta}(t)+\varepsilon p^{\Delta}(t)\right)-L\left(t, x^{\sigma}(t), x^{\Delta}(t)\right)=\varepsilon^{2}\left(p^{\sigma}(t)\right)^{2}+\beta \varepsilon p^{\Delta}(t) .
$$

Hence,

$$
\frac{V(\varepsilon, T)}{\varepsilon}=\inf _{T^{\prime} \geq T} \int_{a}^{T^{\prime}} \frac{\varepsilon^{2}\left(p^{\sigma}(t)\right)^{2}+\beta \varepsilon p^{\Delta}(t)}{\varepsilon} \Delta t=\inf _{T^{\prime} \geq T} \int_{a}^{T^{\prime}}\left(\varepsilon\left(p^{\sigma}(t)\right)^{2}+\beta p^{\Delta}(t)\right) \Delta t .
$$

Choosing $p$ such that $p(a)=0$ and there exists $T_{0}>a$ so that $p(t)=c>0$ for $t \geq T_{0}$, we obtain

$$
\frac{V(\varepsilon, T)}{\varepsilon}=\int_{a}^{T} \varepsilon c^{2} \Delta t+\beta c .
$$

Therefore, assumption 2 of Theorem 4 is violated.

Example 3. Consider the problem

$$
\int_{0}^{+\infty}-\sqrt{1+\left(x^{\Delta}(t)\right)^{2}} \Delta t \longrightarrow \max , \quad x(0)=A .
$$

Since

$$
L\left(t, x^{\sigma}, x^{\Delta}\right)=-\sqrt{1+\left(x^{\Delta}\right)^{2}}
$$

we have

$$
\partial_{3} L=-\frac{x^{\Delta}}{\sqrt{1+\left(x^{\Delta}\right)^{2}}}, \quad \partial_{2} L=0 .
$$

Using the Euler-Lagrange equation (3) we obtain

$$
\tilde{x}^{\Delta}(t)=d \sqrt{1+\left(\tilde{x}^{\Delta}(t)\right)^{2}}, \forall t \in[0,+\infty[
$$

for some $d \in \mathbb{R}$. Solving the latter equation with initial condition $x(0)=A$ we obtain $\tilde{x}(t)=\alpha t+A$, where $\alpha \in \mathbb{R}$. In order to determine $\alpha$ we use the tranversality condition (4), which can be rewritten as

$$
\lim _{T \rightarrow+\infty} \inf _{T^{\prime} \geq T}-\frac{\alpha}{\sqrt{1+\alpha^{2}}}\left(\alpha T^{\prime}+A\right)=0 .
$$

Hence, $\alpha=0$ and $x_{*}(t)=A$ is a candidate to be a maximizer. Observe that

$$
\begin{aligned}
\lim _{T \rightarrow+\infty} \inf _{T^{\prime} \geq T} \int_{0}^{T^{\prime}}\left[L\left(t, x^{\sigma}(t), x^{\Delta}(t)\right)-L\left(t, x_{*}^{\sigma}(t), x_{*}^{\Delta}(t)\right] \Delta t\right. & \\
& =\lim _{T \rightarrow+\infty} \inf _{T^{\prime} \geq T} \int_{0}^{T^{\prime}}\left(1-\sqrt{1+\left(x^{\Delta}(t)\right)^{2}}\right) \Delta t \leq 0
\end{aligned}
$$

for every admissible $x$. Therefore, by Definition $\left[6\right.$ we have that $x_{*}(t)=A$ is indeed the solution to problem (9).

\section{Conclusion and future work}

We considered problems of the calculus of variations on unbounded time scales. Main result provides a new transversality condition. Examples illustrating the application of the new necessary optimality conditions are given in detail. In the particular case $\mathbb{T}=\mathbb{Z}$ our transversality condition gives the discrete time condition obtained by Michel in [28; for the continuous time case, i.e., for $\mathbb{T}=\mathbb{R}$, we obtain the result by Kamihigashi [21. Recently, Okumura et al. 29] generalized the results of Kamihigashi to higher order differential problems. The question of obtaining necessary 
optimality conditions that extend the results of 29. to higher-order infinite horizon problems on time scales remains an interesting open question. While clear that the Euler-Lagrange equations proved in [18, 27. remain valid in the infinite horizon case, the generalization of our transversality condition (4) to higher-order variational problems on time scales is a non-trivial question requiring further investigations.

\section{Acknowledgments}

This work was partially supported by the R\&D unit "Centre for Research on Optimization and Control" (CEOC) of the University of Aveiro, cofinanced by the European Community Fund FEDER/POCI 2010. Agnieszka Malinowska is on leave of absence from Białystok University of Technology (BUT). She was also supported by BUT, via a project of the Polish Ministry of Science and Higher Education "Wsparcie miedzynarodowej mobilnosci naukowcow".

\section{References}

[1] R. Almeida and D. F. M. Torres, Isoperimetric problems on time scales with nabla derivatives, J. Vib. Control 15 (2009), no. 6, 951-958. arXiv:0811.3650

[2] F. M. Atici, D. C. Biles and A. Lebedinsky, An application of time scales to economics, Math. Comput. Modelling 43 (2006), no. 7-8, 718-726.

[3] F. M. Atici and C. S. McMahan, A comparison in the theory of calculus of variations on time scales with an application to the Ramsey model, Nonlinear Dyn. Syst. Theory 9 (2009), no. 1, $1-10$

[4] F. M. Atici and F. Uysal, A production-inventory model of HMMS on time scales, Appl. Math. Lett. 21 (2008), no. 3, 236-243.

[5] Z. Bartosiewicz and D. F. M. Torres, Noether's theorem on time scales, J. Math. Anal. Appl. 342 (2008), no. 2, 1220-1226. arXiv:0709.0400

[6] B. Beavis and Ian. M. Dobbs, Optimization and stability theory for economic analysis, Cambridge Univ. Press, Cambridge, 1990.

[7] M. Bohner, Calculus of variations on time scales, Dynam. Systems Appl. 13 (2004), no. 3-4, 339-349.

[8] M. Bohner, R. A. C. Ferreira and D. F. M. Torres, Integral inequalities and their applications to the calculus of variations on time scales, Math. Inequal. Appl. (2010), in press. arXiv: 1001.3762

[9] M. Bohner and G. Sh. Guseinov, Improper integrals on time scales, Dynam. Systems Appl. 12 (2003), no. 1-2, 45-65.

[10] M. Bohner and A. Peterson, Dynamic equations on time scales, Birkhäuser Boston, Boston, MA, 2001.

[11] M. Bohner and A. Peterson, Advances in dynamic equations on time scales, Birkhäuser Boston, Boston, MA, 2003.

[12] W. A. Brock, On existence of weakly maximal programmes in a multi-sector economy, Review of Economic Studies 37 (1970), 275-280.

[13] D. Cai T. G. Nitta, Treating the future equally: solving undiscounted infinite horizon optimization problems, 2007, arXiv:math/0701371 
[14] D. Cai T. G. Nitta, Constructing the optimal solutions to the undiscounted continuous-time infinite horizon optimization problems, 2008, arXiv:0803.4046

[15] D. Cai T. G. Nitta, Optimal solutions to the infinite horizon problems: Constructing the optimum as the limit of the solutions for the finite horizon problems, Nonlinear Anal. 71 (2009) no. 12, e2103-e2108.

[16] A. C. Chiang, Elements of dynamic optimization, McGraw-Hill, Inc., Singapore, 1992.

[17] P. S. Dasgupta G. M. Heal, Economic theory and exhaustible resources, James Nisbet and Cambridge University Press, Cambridge, 1979.

[18] R. A. C. Ferreira and D. F. M. Torres, Higher-order calculus of variations on time scales, in Mathematical control theory and finance, 149-159, Springer, Berlin, 2008. arXiv:0706.3141

[19] D. Gale, On optimal development in a multisector economy, Rev. Econom. Stud. 34 (1967), $1-19$.

[20] F. Giannessi, A. Maugeri and P. M. Pardalos, Equilibrium problems: nonsmooth optimization and variational inequality methods, Kluwer Acad. Publ., Dordrecht, 2001.

[21] T. Kamihigashi, Necessity of transversality conditions for infinite horizon problems, Econometrica 69 (2001), no. 4, 995-1012.

[22] D. Kravvaritis, G. Pantelides and N. S. Papageorgiou, Optimal programs for a continuous time infinite horizon growth model, Indian J. Pure Appl. Math. 24 (1993), no. 2, 77-86.

[23] S. Lang, Undergraduate analysis, Second edition, Springer, New York, 1997.

[24] A. B. Malinowska and D. F. M. Torres, On the diamond-alpha Riemann integral and mean value theorems on time scales, Dyn. Syst. Appl. 18 (2009), 469-482. arXiv:0804.4420

[25] A. B. Malinowska and D. F. M. Torres, Natural boundary conditions in the calculus of variations, Math. Meth. Appl. Sci. (2010), in press. DOI: 10.1002/mma.1289 arXiv:0812.0705

[26] A. B. Malinowska and D. F. M. Torres, Leitmann's direct method of optimization for absolute extrema of certain problems of the calculus of variations on time scales, Appl. Math. Comput. (2010), in press. DOI: 10.1016/j.amc.2010.01.015 arXiv:1001.1455

[27] N. Martins and D. F. M. Torres, Calculus of variations on time scales with nabla derivatives, Nonlinear Anal. 71 (2009), no. 12, e763-e773. arXiv:0807.2596

[28] P. Michel, Some clarifications on the transversality condition, Econometrica 58 (1990), no. 3, $705-723$.

[29] R. Okomura, D. Cai and T. G. Nitta, Transversality conditions for infinite horizon optimality: higher order differential problems, Nonlinear Anal. 71 (2009), no. 12, e1980-e1984.

[30] P. M. Pardalos, T. M. Rassias and A. A. Khan, Nonlinear analysis and variational problems, Springer, 2010.

[31] F. Ramsey, A mathematical theory of savings, Economic Journal 38 (1928), 543-559.

[32] I. E. Schochetman and R. L. Smith, Optimality criteria for deterministic discrete-time infinite horizon optimization, Int. J. Math. Math. Sci. 2005, no. 1, 57-80.

[33] C. C. Von Weiszacker, Existence of optimal programs of accumulation for an infinite time horizon, Rev. Econom. Stud. 32 (1965), 85-104. 